\title{
Reflets
}

Revue ontaroise d'intervention sociale et communautaire

\section{Deux projets innovateurs destinés aux aînés francophones d'Ottawa-Carleton}

\section{Rachelle Ouellet}

Volume 2, numéro 2, automne 1996

Vieillir à l'aube de l'an 2000

URI : https://id.erudit.org/iderudit/026140ar

DOI : https://doi.org/10.7202/026140ar

Aller au sommaire du numéro

Éditeur(s)

Reflets : Revue ontaroise d'intervention sociale et communautaire

ISSN

1203-4576 (imprimé)

1712-8498 (numérique)

Découvrir la revue

Citer cet article

Ouellet, R. (1996). Deux projets innovateurs destinés aux aînés francophones d'Ottawa-Carleton. Reflets, 2(2), 161-166. https://doi.org/10.7202/026140ar

Tous droits réservés (C) Reflets : Revue ontaroise d'intervention sociale et communautaire, 1996
Ce document est protégé par la loi sur le droit d'auteur. L'utilisation des services d'Érudit (y compris la reproduction) est assujettie à sa politique d'utilisation que vous pouvez consulter en ligne.

https://apropos.erudit.org/fr/usagers/politique-dutilisation/ 


\section{Deux projets innovateurs destinés aux aînés francophones d'Ottawa- Carleton}

\section{Rachelle Ouellet}

Directrice de programme pour les services en français, Conseil sur le vieillissement d'Ottawa-Carleton

\section{Programme pour les aînés francophones des communautés ethno-culturelles}

Dans la région d'Ottawa-Carleton, la réalité multiculturelle au sein d'une population généralement vieillissante est très présente. Les principales communautés ethniques francophones sont composées d'Africains, d'Haïtiens, de Libanais et de Vietnamiens (Beaulieu et al., 1994). On trouve environ 1300 personnes âgées appartenant à divers groupes ethniques qui parlent français (Racine, 1994).

Par ailleurs, on remarque que les personnes âgées francophones des communautés ethno-culturelles utilisent moins les services offerts par les organismes communautaires que ne le font les aînés francophones de souche. Diverses raisons semblent expliquer cette situation, en particulier les barrières d'ordre culturel incluant la difficulté à lire ou à écrire le français, le manque d'information sur les services disponibles, la situation économique précaire, ainsi que des obstacles d'ordre systémique. 
Au début de l'année 1995, l'Assemblée des affaires francophones $\mathrm{du}$ Conseil sur le vieillissement d'Ottawa-Carleton a élaboré un projet visant à mettre sur pied, au sein des trois centres pour ainés francophones de la région, un programme d'activités à caractère socio-récréatif et culturel pour les membres des communautés ethno-culturelles. Grâce à une subvention du ministère de la Culture, du Tourisme et des Loisirs de l'Ontario, destinée à la création de loisirs communautaires, ce projet d'une durée d'un an a pu voir le jour.

La participation à des activités de loisirs favorise une vie plus saine et productive, stimule la fierté locale et procure un sens de réussite personnelle et d'appartenance, tout en permettant de briser l'isolement. Par conséquent, afin de remédier au manque de participation des aînés francophones appartenant aux divers groupes ethniques, des activités socio-récréatives et culturelles sont organisées régulièrement dans les centres de jour francophones de la région.

Les activités sont très variées: conférences sur des thèmes divers, jeux de société, artisanat, dîners-causerie, visites et sorties de groupe, etc. Le recrutement des participants et participantes se fait par le biais des différentes associations de groupes ethniques francophones de la région. Dépliants et affiches sont préparés et distribués par ces associations. Il importe de souligner leur remarquable collaboration, ainsi que celle des centres de jour, ce qui explique le succès que connait ce projet. Le taux de participation est en effet très satisfaisant et les commentaires des participants sont positifs.

Il faut souligner que les membres réguliers des centres de jour sont également conviés à participer aux différentes activités organisées par la coordonnatrice, car le projet a pour but éventuel de favoriser progressivement le rapprochement des personnes âgées des communautés ethno-culturelles avec les aînés francophones de souche, en vue de consolider l'appartenance à la société d'accueil, d'améliorer les connaissances du français et de briser les stéréotypes entretenus de part et d'autre.

Le seul fait de sensibiliser les aînés francophones des communautés ethno-culturelles aux bienfaits des contacts sociaux et des 
activités de loisirs représente un pas en direction de l'objectif ultime. De plus, l'information sur les services disponibles et la façon de se les procurer est un élément essentiel qui facilite les liens avec la communauté d'accueil. Bien entendu, les besoins des aînés francophones des communautés ethniques vont au-delà des activités socio-récréatives et culturelles; l'Assemblée des affaires francophones, on pense déjà à la possibilité d'élargir ce projet pilote pour tenter de mieux répondre à ces autres besoins. D'autres sources de financement devront être identifiées pour assurer un suivi à cette entreprise.

\section{Projet pilote «Vieillir chez soi»}

Ce projet pilote est né à la suite d'une étude faite en 1991-92 par un groupe de consultants pour le Conseil sur le vieillissement d'Ottawa-Carleton (CSv) et le Logement Ottawa-Carleton (LOC), en vue d'identifier les moyens permettant aux personnes âgées de demeurer le plus longtemps possible dans leur communauté, et d'éviter les cas inopportuns de placement en établissement.

Au cours de l'étude, un modèle a été élaboré en vue de coordonner les services sociaux et de santé fournis aux résidents des logements pour personnes âgées de LOC, tout en répondant aux besoins relevés par les résidents, et pour leur permettre de «Vieillir chez soi».

- le modèle doit répondre à des besoins reconnus se rapportant principalement à quatre grands domaines: information et orientation, sécurité des personnes et des biens, écoute et réconfort, et relations entre locataires;

- le modèle doit promouvoir l'entraide et favoriser l'autonomie des locataires, en les aidant à résoudre leurs problèmes, aussi bien sur le plan individuel que collectif, par l'entremise de l'association des locataires;

- le modèle doit permettre aux associations de locataires de prendre des décisions et de les appliquer dans l'intérêt de toutes les personnes 
vivant dans les immeubles, du fait qu'un plus grand nombre de personnes de l'immeuble s'intéresse aux activités de l'association des locataires et y participe;

- le modèle doit aider les locataires à "vieillir chez soi», en améliorant leur qualité de vie, en reconnaissant et en aidant ceux et celles à qui les problèmes de santé ou d'isolement social font courir le risque d'un placement en établissement de soins de longue durée.

Le modèle comporte quatre éléments :

1) postes de coordination du soutien aux personnes âgées,

2) services sur place et centres de ressources,

3) équipe de soins à domicile pour les clients admissibles et,

4) comités conjoints de travailleurs et de locataires.

Depuis plus d'un an à Ottawa-Carleton, dans trois immeubles de logements pour personnes âgées, certaines composantes de ce modèle sont actuellement à l'essai. Les éléments appliqués dans chaque immeuble correspondent aux besoins exprimés et aux ressources disponibles. Une douzaine d'organismes, dont le Conseil sur le vieillissement, participent activement au fonctionnement de ces projets pilotes.

L'un d'eux se déroule dans un immeuble de logements dont les résidents sont en majorité francophones. Les membres du comité de fonctionnement sont des intervenants de différents organismes servant les aînés, ainsi que des représentants des résidents de l'immeuble; ils se rencontrent donc chaque mois pour établir des stratégies visant à aider chaque personne à maintenir un mode de vie autonome et à améliorer son bien-être.

C'est ainsi qu'avec l'aide de bénévoles, un centre de ressources et de renseignements à été mis sur pied, rassemblant l'information sur les différents services destinés aux personnes ainées de la région d'Ottawa-Carleton. Des personnes-ressources sont également disponibles sur place à raison d'une dizaine d'heures par semaine, et assistent les résidents dans leurs recherches. Les échanges qui prennent place lors des visites au centre de ressources permettent aux personnes-ressources d'être attentives aux préoccupations et aux suggestions des résidents, particulièrement 
au niveau de leurs besoins en services. Tout ceci est soumis au comité de fonctionnement, en vue d'identifier des moyens d'améliorer en conséquence le système de renseignements.

On prépare un calendrier d'activités organisées dans la salle polyvalente de l'immeuble, et auxquelles les résidents seront invités à assister. La collaboration active de l'Association des locataires de l'immeuble est essentielle, car c'est elle la mieux placée pour connaître les besoins et les attentes des résidents concernant ce projet pilote.

Dans un deuxième temps, un local sera réservé aux travailleurs et travailleuses de première ligne qui fournissent des services aux résidents de l'immeuble, ce qui facilitera grandement la coordination, la communication et la prestation des services. Cet aspect du projet pilote reste encore à développer dans cet immeuble. Mais on travaille actuellement à faciliter les échanges et la transmission d'information entre les intervenants et intervenantes qui œuvrent auprès des aînés habitant l'immeuble.

S'il est concluant, ce projet pilote sera certainement l'un des moyens d'aider les personnes âgées à demeurer à leur domicile le plus longtemps possible. Il semble que c'est ce que souhaitent de nombreuses personnes âgées francophones de la région d'OttawaCarleton, selon la récente étude réalisée par une firme de consultants pour l'Assemblée des affaires francophones du Conseil sur le vieillissement (Lemire, 1995). À l'aube de l'an 2000, étant donné les changements rapides et nombreux qui s'opèrent au niveau des services sociaux et de santé, ce type d'initiative vaut certainement la peine d'être étudié de près.

\section{Bibliographie}

BEAULIEU, Jocelyne, André NADEAU et al., (1994). Intégration des communautés ethno-culturelles. Ottawa, Beaulieu, Nadeau et al.

CONSEIL SUR LE VIEILLISSEMENT - OTTAWA-CARLETON (1992). Création d'un modèle de coordination de services et d'aide aux locataires destiné aux personnes âgées. Ottawa, Conseil sur le vieillissement - Ottawa-Carleton. 
LEMIRE, D., (1995). les nouvelles avenues en matière de logement chez les personnes francophones de 55 ans et plus de la région d'Ottawa-Carleton. Beaulieu \& Lemire, consultation, recherches et formation. Ottawa.

RACINE, J.L., (1994). Nombre de personnes âgées de 65 ans et plus ayant le français comme langue parlée. Document ronéotypé, Ottawa. 